University of New Hampshire

University of New Hampshire Scholars' Repository

Space Science Center

Institute for the Study of Earth, Oceans, and

Space (EOS)

10-19-1999

\title{
Recent progress in the development of a solar neutron tracking device (SONTRAC)
}

James M. Ryan

University of New Hampshire, James.Ryan@unh.edu

Wenhui Li

University of New Hampshire - Main Campus

John R. Macri

University of New Hampshire - Main Campus, John.Macri@unh.edu

Mark L. McConnell

University of New Hampshire - Main Campus, mark.mcconnell@unh.edu

Carlos Castaneda

University of California - Davis

See next page for additional authors

Follow this and additional works at: https://scholars.unh.edu/ssc

Part of the Astrophysics and Astronomy Commons

\section{Recommended Citation}

James M. Ryan ; Wenhui Li ; John R. Macri ; Mark L. McConnell ; Carlos M. Castaneda and Juan L. Romero "Recent progress in the development of a solar neutron tracking device (SONTRAC)", Proc. SPIE 3768, Hard X-Ray, Gamma-Ray, and Neutron Detector Physics, 496 (October 19, 1999); doi:10.1117/12.366621; http://dx.doi.org/10.1117/12.366621

This Conference Proceeding is brought to you for free and open access by the Institute for the Study of Earth, Oceans, and Space (EOS) at University of New Hampshire Scholars' Repository. It has been accepted for inclusion in Space Science Center by an authorized administrator of University of New Hampshire Scholars' Repository. For more information, please contact Scholarly.Communication@unh.edu. 


\section{Authors}

James M. Ryan, Wenhui Li, John R. Macri, Mark L. McConnell, Carlos Castaneda, and Juan L. Romero 


\title{
Recent progress in the development of a solar neutron tracking device (SONTRAC)
}

\author{
James M. Ryan ${ }^{*}$, Wenhui Li ${ }^{\mathrm{a}}$, John R. Macri ${ }^{\mathrm{a}}$, Mark L. McConnell ${ }^{\mathrm{a}}$ \\ Carlos M. Castaneda ${ }^{\mathrm{b}}$, Juan L. Romero ${ }^{\mathrm{b}}$ \\ ${ }^{a}$ Space Science Center, University of New Hampshire, Durham, NH 03824 \\ ${ }^{\mathrm{b}}$ Crocker Nuclear Laboratory, University of California, Davis CA 95616
}

\begin{abstract}
We report the results of recent calibration data analysis of a prototype scintillating fiber tracking detector system designed to perform imaging, spectroscopy and particle identification on 20 to $250 \mathrm{MeV}$ neutrons and protons. We present the neutron imaging concept and briefly review the detection principle and the prototype description. The prototype detector system records ionization track data on an event-by-event basis allowing event selection criteria to be used in the off-line analysis. Images of acrylic phantoms from the analysis of recent proton beam calibrations (14 to $65 \mathrm{MeV}$ range) are presented as demonstrations of the particle identification, imaging and energy measurement capabilities. The measured position resolution is $<500 \mu \mathrm{m}$. The measured energy resolution $(\triangle \mathrm{E} / \mathrm{E}, \mathrm{FWHM})$ is $14.2 \%$ at $35 \mathrm{MeV}$.

The detection techniques employed can be applied to measurements in a variety of disciplines including solar and atmospheric physics, radiation therapy and nuclear materials monitoring. These applications are discussed briefly as are alternative detector configurations and future development plans.
\end{abstract}

Keywords: neutron, proton, tracking, imaging, spectroscopy, scintillating fiber, CCD, image intensifier, solar

\section{INTRODUCTION AND MOTIVATION}

Neutron telescopes based on double scatters are particularly effective in high background environments. ${ }^{1,2}$ The neutron telescope described here, known as SONTRAC, the SOlar Neutron TRACking telescope, is under development to study the high energy processes associated with solar flares. ${ }^{3}$ When high-energy charged particle reactions occur on the surface of the Sun, neutrons carry away information about the spectrum of ions that produced them and can be used as diagnostic measures of that spectrum. ${ }^{4}$

A number of other applications for such a device have also been identified. In the earth's atmosphere neutrons above 10 $\mathrm{MeV}$ produce so-called soft error upsets in microcircuitry and they also present a radiation health hazard for personnel at high altitudes. $^{5,6,7}$ Neutron telescopes can accurately determine the properties of the neutron background. Neutron tracking detectors can also be employed to accurately locate nuclear materials (waste, spills).

The success of Proton radiotherapy is based on the precision with which the dose is deposited in the tumor volume. ${ }^{8}$ The tracking detector described here can be used to directly detect incident protons and precisely image the absorbing material to properly register a patient within the proton beam.

\section{NEUTRON IMAGING CONCEPT}

The detector measures the energy and direction of neutrons by detecting double neutron-proton scatters and recording images of the ionization tracks of the recoil protons in a densely packed bundle of scintillating plastic fibers stacked in orthogonal layers. The kinematics of the scatter are determined by tracking the recoil protons.

The double-scattering of a non-relativistic neutron in a solid block of plastic scintillator is illustrated in Figure 1. Neutrons interact in plastic scintillator either by elastically scattering from hydrogen (n-p) or by interacting with carbon (n-C). The n-p events are the most useful. For the non-relativistic case,

* Correspondence: E-mail: James.Ryan@unh.edu; Telephone: 603 862-3510; FAX: 603 862-4685 


$$
\sin ^{2} \phi_{n^{\prime}}=\cos ^{2} \phi_{p^{\prime}}=\frac{E_{p^{\prime}}}{E_{n^{\prime}}+E_{p^{\prime}}}=\frac{E_{p^{\prime}}}{E_{n}}
$$

where $E_{n}$ is the incident neutron energy; $E_{n}$, and $E_{p}$, are the scattered neutron and photon energies, respectively; $\phi_{n}$ ' and $\phi_{p}$, are the neutron and proton scatter angles, respectively. The kinematics of nonrelativistic scattering further dictate that the scattered neutron and proton momenta are perpendicular to one another.

If the incident direction of a neutron is known, then the measurement of the energy and direction of a recoil proton in a single scatter is sufficient to determine the incident neutron energy. In particular, if the incident direction is known, then $\phi_{n}$, is determined and the neutron energy is

$$
E_{n}=\frac{E_{p^{\prime}}}{\cos ^{2} \phi_{p^{\prime}}}
$$

A more general approach, however, is provided by double-scatter events (Figure 1). If both recoil protons in a double scatter event are measured, then the energy and incident direction of the neutron are uniquely determined. The angular and energy
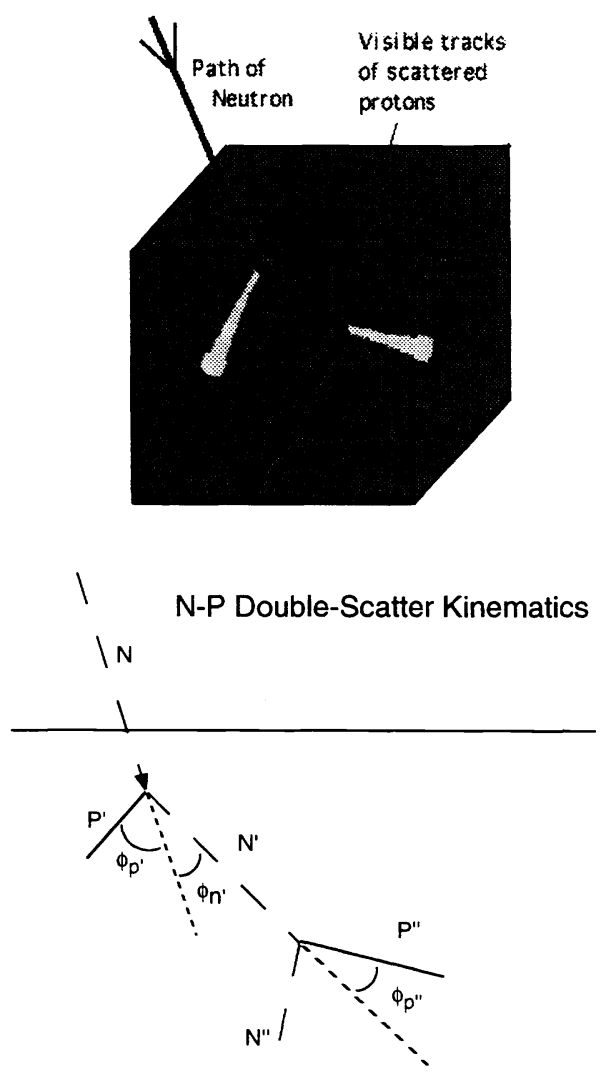

Figure 1. Schematic of non-relativistic double scatter neutron event in a block of plastic scintillator. resolution depend upon the ability to precisely measure the energy and direction of the recoil protons. Double scatter events can be used to measure neutron intensity from an extended source such as the secondary neutrons produced in the atmosphere by the primary cosmic radiation. For solar neutrons, double scatter events are also preferred, because they allow for a more complete separation of the source signal from the ambient background.

\section{DETECTOR CONCEPT}

The tracking detector employs a closely packed bundle of square cross section plastic-scintillator fibers. The fibers are arranged in stacked planes with the fibers in each plane orthogonal to those in the planes above and below. This alternating orientation allows one to record stereoscopic images and track ionizing particles in three dimensions in the scintillating fiber bundle. We have baselined a fiber pitch of $300 \mu \mathrm{m}$, a value chosen so that a $10 \mathrm{MeV}$ proton traverses several fibers before stopping. Figure 2 represents a tiny $(3 \mathrm{~mm}$ cubic) segment of such a bundle. The neutron angular resolution is dependent upon the ability to precisely track the recoil protons. Track imaging employs two CCD cameras, one for each set of orthogonal fiber planes. The ionization track length is a sensitive measure of the recoil proton energy. The location of the Bragg peak, which corresponds to the greater ionization at the end of the track, fixes the track direction.

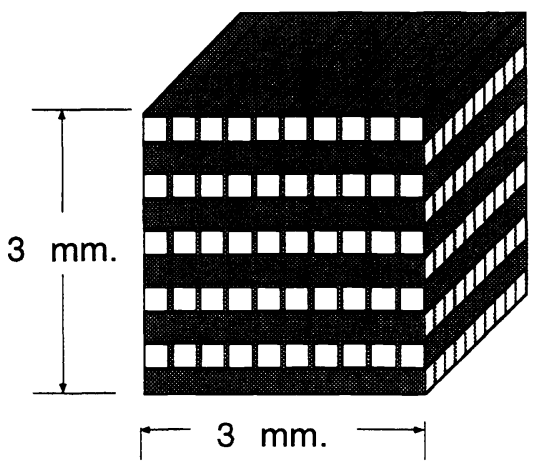

Figure 2. Small segment of a fiber bundle, illustrating orthogonal scintillator fiber layers with $300 \mu \mathrm{m}$ pitch. 


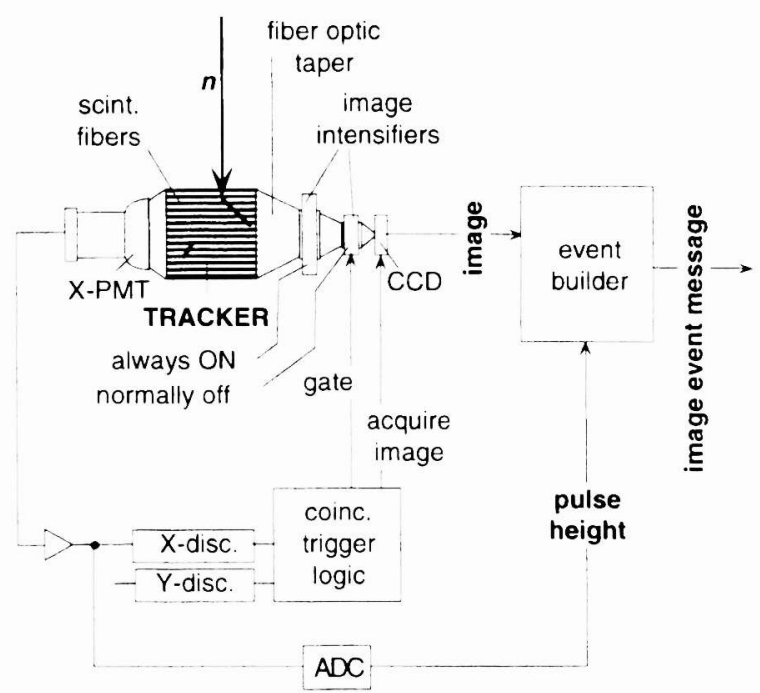

Figure 3. Functional diagram illustrating the detector concept and the signal processing logic.

\section{PROTOTYPE DESCRIPTION}

The first SONTRAC laboratory prototype is shown in Figure 4. The prototype tracker is a $10 \mathrm{~cm}$ long bundle of $250 \mu \mathrm{m}$ square scintillating plastic fibers on $300 \mu \mathrm{m}$ pitch within a $12.7 \mathrm{~mm}$ square envelope. It is limited to tracking in two dimensions. The specifications of its key components and the manner in which it is operated (fiber composition and pitch, photocathode and phosphor composition, self triggered image gating and acquisition scheme) have been reported. ${ }^{9}$

\section{MEASUREMENTS AND ANALYSIS}

\subsection{Neutron detection demonstration}

The SONTRAC prototype was exposed to $14 \mathrm{MeV}$ neutrons at San Diego State University and to higher energy (up to $65 \mathrm{MeV}$ ) neutrons and to protons at the Crocker Laboratory cyclotron facility at the University of California at Davis. Figure 5 shows a raw CCD image of a neutron double interaction in the prototype fiber bundle. Two recoil proton tracks are evident from a single neutron $(\sim 65 \mathrm{MeV})$ incident from the top of the figure. Note the evidence of the Bragg peak. "Holes" in the track images represent the passage of the ionizing particles through the passive cladding and EMA materials.
A functional diagram of an experiment utilizing the SONTRAC concept is shown in Fig. 3. The detector's spectroscopic, track detection and imaging components cover the entire light emitting area of the scintillating fiber bundle and are duplicated in the orthogonal dimension (not shown). The scintillation light signal is collected and processed at both ends of the fiber bundle. At one end a signal above threshold from a photomultiplier tube (PMT) fires a discriminator that in turn provides a signal to the trigger logic circuitry. At the other end of the fiber bundle. fiberoptic tapers and a pair of image intensifiers demagnify. capture and hold the scintillation-light image of the ionization track(s) for readout by the CCD camera. The first image intensifier in this chain is always $O N$. Its phosphor holds the image for approximately $1 \mathrm{~ms}$. The second image intensifier in this chain is normally in the gated-OFF condition and no image signal is passed to the CCD sensor. However, when the trigger logic registers the proper coincidence, the track image and PMT pulse height data are acquired and passed to an event builder and combined with auxiliary information for subsequent event-byevent analysis.

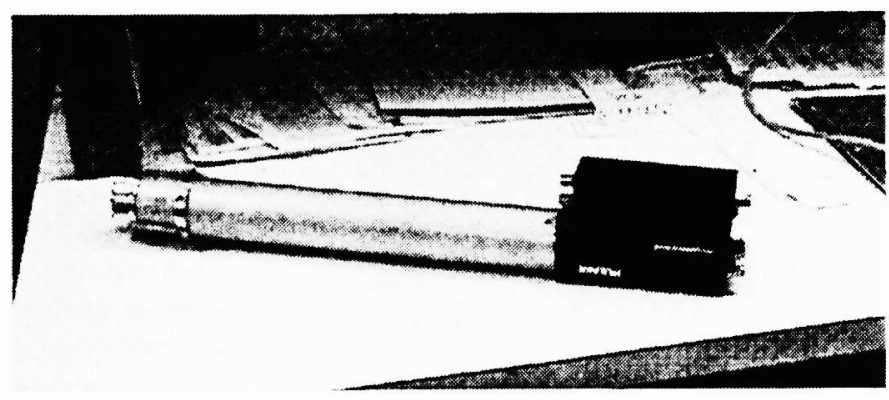

Figure 4. The SONTRAC prototype detector. The (CD) camera is shown to the right and the PMT to the left. 


\subsection{Measurements with protons}

A practical calibration approach involves direct irradiation of the fiber bundle with protons. Figure 6 shows the track of a $\sim 20 \mathrm{MeV}$ proton incident from the left. In this figure the intensity is averaged over each fiber and a calibration mask of the fiber bundle is superposed on the track image. Note that the track of the incident proton, unlike those from incident neutrons, starts at the edge of the bundle.

Measurements performed at the Crocker Laboratory used a variety of phantom absorbers placed between the proton beam and the tracking detector to measure and calibrate the response and to demonstrate its spectroscopic and imaging capabilities. The geometry for one such test is illustrated in Figure 7. The phantom absorber is a $24.78 \mathrm{~mm}$ thick acrylic block $\left(1.18 \mathrm{~g} / \mathrm{cm}^{3}\right)$ with 3.88 mm deep, $2.86 \mathrm{~mm}$ wide slots on $6 \mathrm{~mm}$ centers. The slots run parallel to the scintillating fiber axis. The prototype's scintillating fiber bundle is $12.7 \mathrm{~mm}$ wide (42 fibers on $0.3 \mathrm{~mm}$ pitch) thus spanning slightly more than two pitch lengths of the phantom pattern.

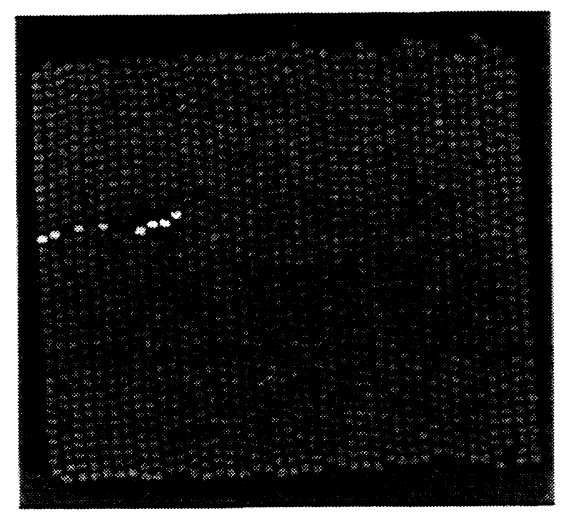

Figure 6. Track of $\sim 20 \mathrm{MeV}$ proton incident from the left.

Figure 8 shows the accumulated sum of intensity per fiber for the track images of 555 selected events. Recall that data are recorded for every event permitting analysis on a variety of criteria. In this case particles scattered from nearby material in the laboratory were identified and rejected either by the oblique slope of the track image or by the point of entry into the fiber bundle. The figure illustrates the signature of two pitch cycles of the phantom slot pattern.

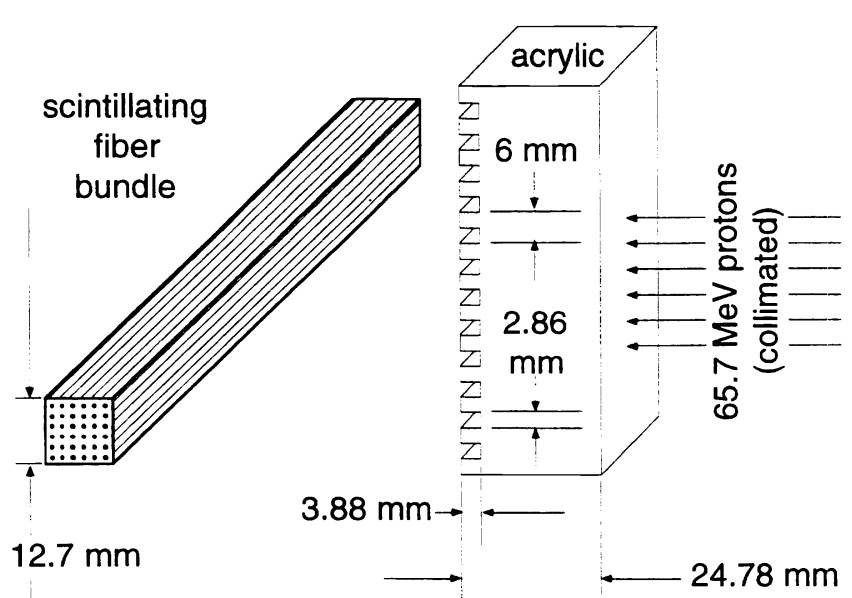

Figure 7. Test geometry for phantom imaging.

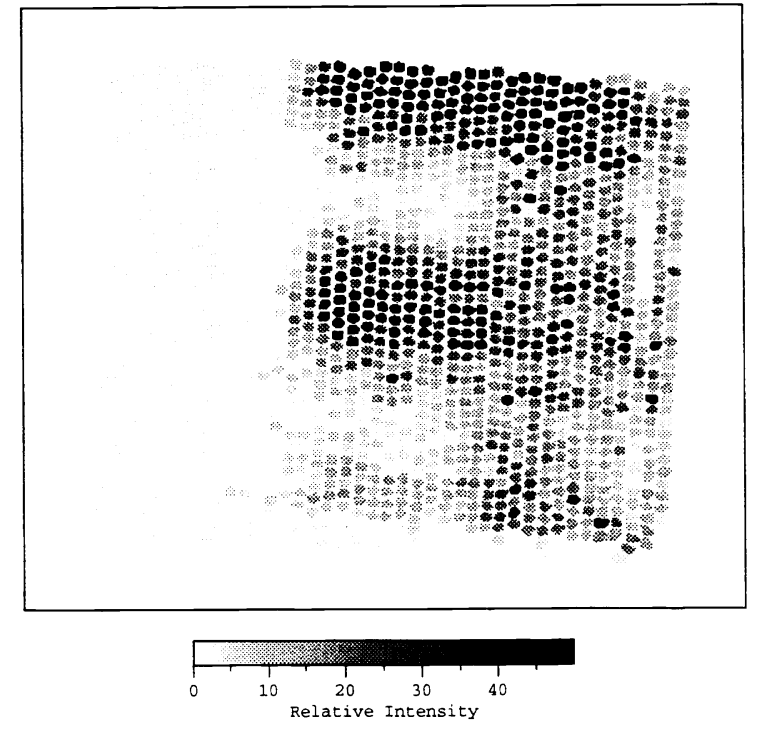

Figure 8. Image of slotted phantom accumulated from proton track images. Protons are incident from the right. 
Figure 9 is the distribution of the computed track length of each event represented in Figure 8. The sharp peaks represent individual fibers. Two Gaussian envelopes are evident representing the two proton energies emerging from the slotted phantom.

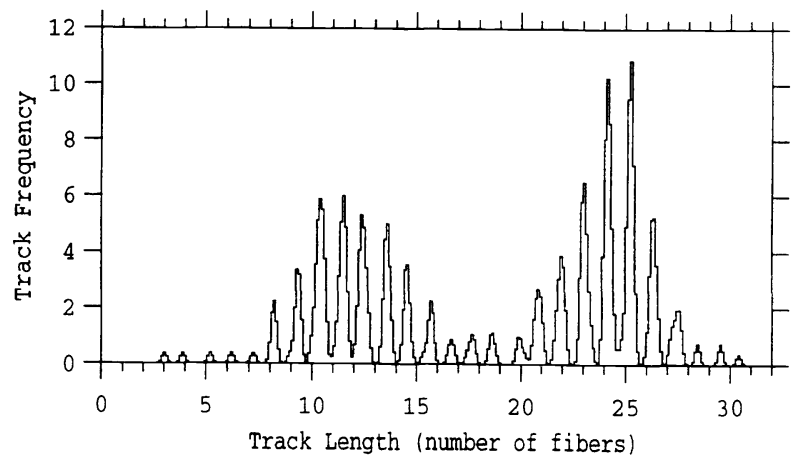

Figure 9. Distribution of computed track length for the 555 events represented in the Figure 8.

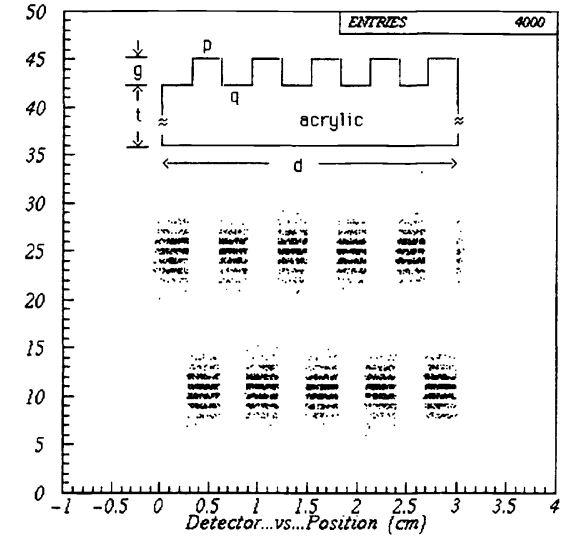

Figure 10. Simulation of proton range for slotted phantom measurements. Incident proton energy $65.7 \pm$ $0.3 \mathrm{MeV}$.

The measured track length is $24.5 \pm 1.3$ fibers for protons passing through the slots and $11.8 \pm 1.3$ fibers for protons passing through the full thickness of the acrylic block. The difference of $3.8 \pm 0.5 \mathrm{~mm}(12.7 \pm 1.8$ fibers $)$ agrees with the depth of the slots in the phantom. These data agree well with simulations that include straggling (Figure 10), which predict track lengths of $25.3 \pm 1.3$ fibers and $11.0 \pm 1.4$ fibers, respectively. The measured energy resolutions $(\Delta \mathrm{E} / \mathrm{E}, \mathrm{FWHM})$ are $14.2 \%$ at 35 $\mathrm{MeV}$ and $16.5 \%$ at $27 \mathrm{MeV}$. 

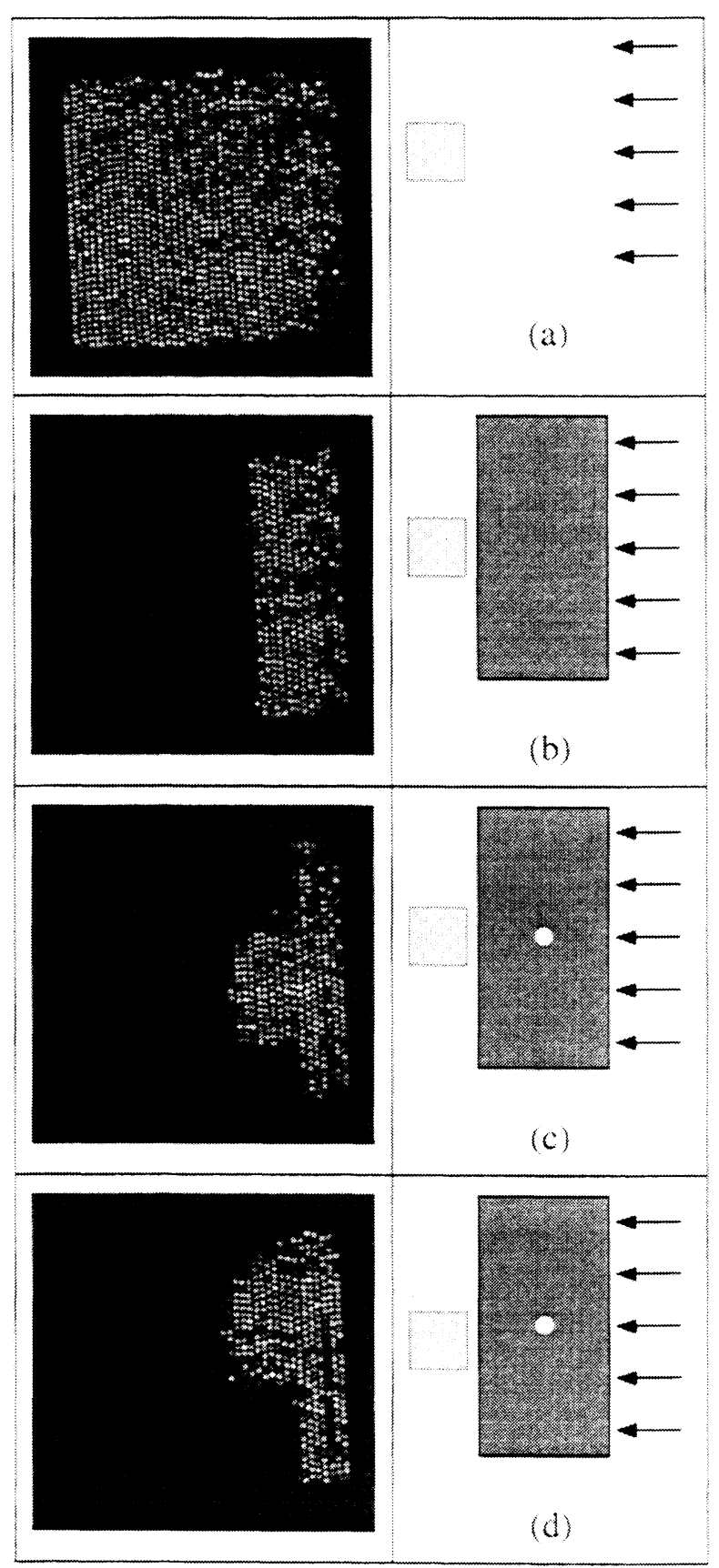

Figure 11. Relative intensity of accumulated track images (left). The proton beam was passed through a variety of acrylic phantoms (right).
Images from other runs employing other acrylic phantom geometries are shown in Figure 11. The $65.7 \mathrm{MeV}$ protons pass completely through the fiber bundle with no phantom absorber present (a). Uniform proton track lengths are observed in the fiber bundle detector when a phantom of uniform thickness is placed between it and the proton beam (b). Longer proton track lengths corresponding to lower phantom density are observed when a uniform thickness phantom with a $3 \mathrm{~mm}$ diameter hole drilled parallel to the fiber axis is used (c). The position of the hole in the image shifts accordingly when the detector is moved $3 \mathrm{~mm}$ vertically (d).

\section{FUTURE WORK}

On-going work involves the fabrication and test of a minimally-configured three-dimensional SONTRAC science model (SM). Unlike the first SONTRAC prototype described above, the science model is based on a fiber bundle with orthogonal fiber layers (as depicted in Figure 2), thus permitting track imaging and reconstruction in 3 dimensions. The SM will employ a $5 \times 5 \times 5 \mathrm{~cm}$ scintillating plastic fiber tracking detector with appropriately sized optoelectronic components (Figure 12). The larger size of the SM fiber bundle will permit measurements at higher energies where $n-C$ scatters occur. The goals of this science model study are to characterize the performance of the three dimensional version of SONTRAC and to refine the necessary electronics configuration and data processing algorithms for an engineering model. Calibrations are planned for both neutrons and protons at energies from $10-80 \mathrm{MeV}$, a range which covers from below the nominal threshold up to energies where neutron double scatter events are no longer contained within the SM fiber block.

The small $(300 \mu \mathrm{m})$ fiber size of the SONTRAC design dictates the use of a CCD-based system for reading out the signals from the scintillating fiber bundle. However, we will be closely monitoring technology developments that may offer significant advantages in the readout of a SONTRAC device. Readout schemes based on the use of multi-anode PMTs, hybrid PMTs or on avalanche photodiode arrays (APDs) may become viable options within the next few years.

The operation of a SONTRAC device represents the accumulation of large volumes of (imaging) data. For remote operations, much of the processing should take place on-board to reduce telemetry and/or data recording requirements. The science model study represents an opportunity to investigate data processing issues important in future engineering and
processing algorithms for (three-dimensional) event recognition, flight model detectors. We will develop and evaluate data processing algorithms for (three-dimensional)
track recognition, data compression and track reconstruction that could be employed in future instrumentation. 


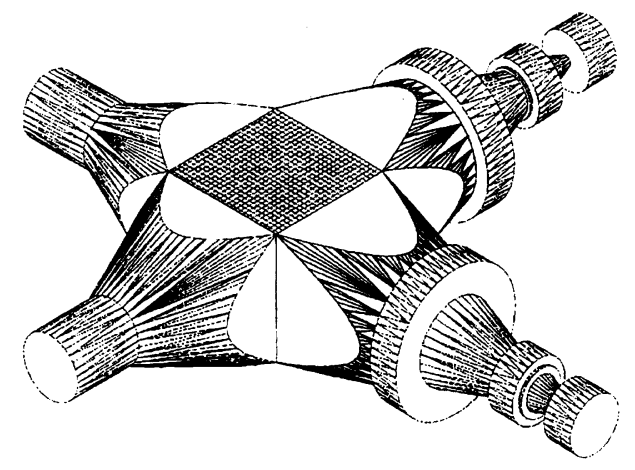

Figure 12. Conceptual sketch of a $5 \times 5 \times 5 \mathrm{~cm}$ SONTRAC science model.

\section{CONCLUSIONS}

This technique works! The signal to noise ratio is at least four times higher than required. There is sufficient signal even in the more demanding applications where detection of the tracks of minimum ionizing particles is required. ${ }^{9}$ Event-by-event detection and measurement of track length (energy), track direction and particle type has been accomplished. Specifically, we have fabricated a small SONTRAC prototype and demonstrated a self-triggered detector system for ionization track imaging of individual events. This has been done for minimum ionizing cosmic ray muons, the recoil protons from incident $14 \mathrm{MeV}$ to $65 \mathrm{MeV}$ neutrons and for incident $20 \mathrm{MeV}$ to $65 \mathrm{MeV}$ protons. We have demonstrated SONTRAC's neutron detection, spectroscopic and imaging capabilities from the threshold necessary for the solar physics study. We have explored and developed useful calibration and data analysis techniques using proton beams and in the process we have demonstrated imaging capabilities directly applicable to other fields. We have developed a preliminary response model and demonstrated agreement with the laboratory measurements. As a result of this effort key engineering parameters such as photoelectron yield, achievable energy threshold, scintillating fiber composition, fiber pitch, EMA requirements, photocathode and phosphor selection have been determined allowing us now to extend these techniques to larger detectors for higher energies with the ability to track in three dimensions.

\section{ACKNOWLEDGEMENTS}

This work is supported under a grant from NASA's Space Physics Supporting Research and Technology (SR\&T) program.

\section{REFERENCES}

1. G.Kanbach, et al., J. Geophys. Res., vol. 79, p 5159, 1974.

2. J. M. Ryan, et al., in Data Analysis in Astronomy IV, p 261, Plenum Press, NY, 1992.

3. C. B. Wunderer, D. Holslin, J. R. Macri, M. L. McConnell, J. M. Ryan, "SONTRAC - A Low Background, Large Area Solar Neutron Spectrometer," Workshop Record, Conference on the High Energy Radiation Background $n$ Space, IEEE Nuclear and Space Radiation Effects Conference, Snowmaass, Colorado, p 73, 1997

4. H. Hudson and J. Ryan. Annual Review Astronomy and Astrophysics, vol. 33, pp. 239-282, 1995.

5. M. Walt, Introduction to Geomagnetically Trapped Radiation, Cambridge University Press, 1994.

6. T. O'Gorman, et al., IBM Journal of Research and Development, vol. 40, No. 1, p. 41, 1996.

7. J. M. Ryan and R. Saxena, "Ground Level Neutron Measurements from 10-170 MeV", Proc. Amer. Nucl. Soc. Topical Meetings, Radiation Protection and Shielding, vol. 1, pp. 219-226, 1996.

8. J. L. Romero, et al., "Patient positioning for protontherapy using a proton range telescope", Nuclear Instruments and Methods in Physics Research A, vol. 356, pp. 558-565, 1995.

9. J.M. Ryan, et al., "A prototype for SONTRAC, a scintillating plastic fiber detector for solar neutron spectroscopy",SPIE Proceedings 3114, pp. 514-525, 1997 